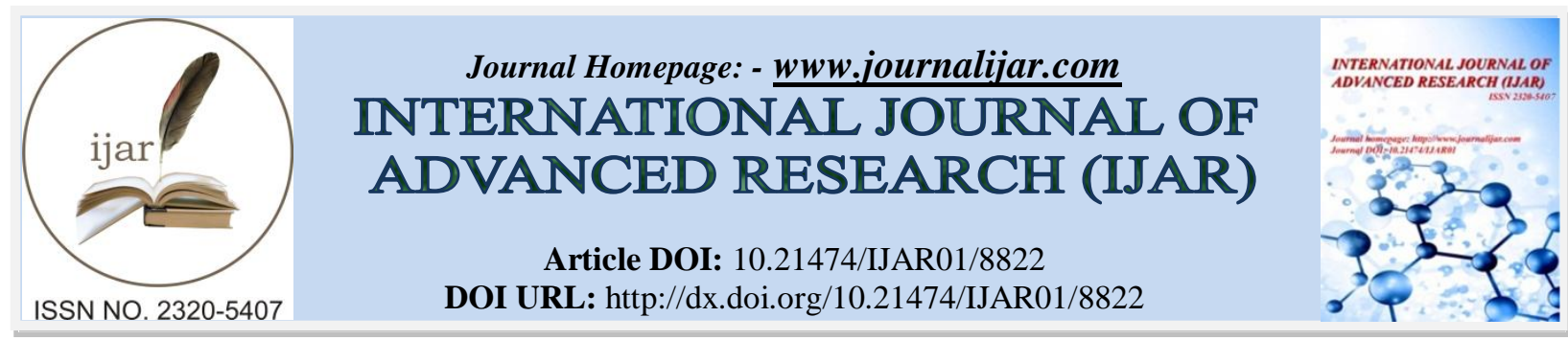

CASE REPORT ARTICLE

\title{
PRESURGICAL NASOALVEOLAR MOLDING ASSISTED PRIMARY RECONSTRUCTION IN 5-DAY OLD INFANT WITH UNILATERAL CLEFT LIP, ALVEOLUS AND PALATE USING PSIO-J HOOK APPLIANCE - A CASE REPORT.
}

\author{
Justin Jolly ${ }^{1}$, Goutham Reddy ${ }^{2}$, Sunil Muddaiah ${ }^{3}$, Sanju Somaiah ${ }^{3}$, Balakrishna Shetty ${ }^{3}$ and Roopa $\mathrm{S}^{4}$ \\ 1. MDS- Post Graduate Student, Department of Orthodontics, Coorg Institute of Dental Sciences, Coorg, \\ Karnataka, India. \\ 2. Professor and Head, Department of Orthodontics, Coorg Institute of Dental Sciences, Coorg, Karnataka, India. \\ 3. Professor, Department of Orthodontics, Coorg Institute of Dental Sciences, Coorg, Karnataka, India. \\ 4. Senior lecturer, Department of Orthodontics, Coorg Institute of Dental Sciences, Coorg, Karnataka.
}

\section{Manuscript Info}

Manuscript History

Received: 05 February 2019

Final Accepted: 07 March 2019

Published: April 2019

Key words:

Presurgical infant orthopedic (PSIO), cleft lip, cleft palate, cleft alveolus, pre-surgical nasoalveolar molding, PSIO-J hook appliance.

\section{Abstract:}

Introduction: Management of cleft patients is quite challenging and controversial. Advances in reconstructive surgery have significantly improved the quality of repair for cleft of the lip, alveolus and palate. This case report describes a new approach of presurgical nasoalveolar molding (PNAM) therapy in a 5 day old infant using PSIO-J hook appliance.

Methods: Lip taping was started on the initial visit using 3M SteriStrip. Initial impressions were made to fabricate the PSIO appliance. On second visit appliance was delivered to approximate the cleft alveolus. Once the cleft gap has been reduced to approximately $5 \mathrm{~mm}$ a J- hook modification was given to avoid bulkiness and thereby enhancing retention. Conventional nasal stent exerts a reciprocal intraoral molding force against the alveolar segments hence this drawback was eliminated by using J-hook modification appliance. Following surgical repair of lip, the lip was taped for several weeks and the use of PSIO-J hook appliance was discontinued.

Results: There was significant improvement in nasal tip projection, columella size on cleft side and position of lower lateral cartilages as well as approximation of the lip segments. Additionally the alveolar defect reduced from $14 \mathrm{~mm}$ to $3 \mathrm{~mm}$ from pre NAM to post NAM.

Conclusion: The pre-surgical nasoalveolar molding assisted primary reconstruction using PSIO-J hook appliance results is an overall improvement in the esthetics of the nasolabial complex in cleft conditions while minimizing the extent of surgery and the overall number of surgical procedures.

Copy Right, IJAR, 2019,. All rights reserved.

\section{Introduction:-}

Management of cleft patients is challenging in the world of orthodontics. Advances in reconstructive surgery have significantly improved the quality of repair for cleft of the lip, alveolus and palate. The basic goal of any approach

Corresponding Author:- Justin Jolly.

Address:- MDS- Post Graduate Student, Department of Orthodontics, Coorg Institute of Dental Sciences, 
for unilateral or bilateral cleft lip, alveolus and palate repair is to produce harmonious facial esthetics and normal anatomy. ${ }^{1,2}$

Ideally, deficient tissues should be expanded and malpositioned structures should be repositioned prior to surgical correction which provides a foundation for a less invasive surgical repair. Historically, the use of presurgical infant orthopedic (PSIO) appliances, or molding plate therapy, has aided significantly in reducing the size of clefts of the alveolus and hard palate prior to surgery. ${ }^{3}$ Since its introduction by McNeil in 1950, various techniques for molding the intraoral alveolar segments closer together in unilateral and bilateral cleft situations have been described (Mylin1968, Latham-1980). Although controversy surrounds the use of PSIO devices in conjunction with various surgical protocols when employed to move only the alveolar segments closer together, this case report describes a new approach of presurgical nasoalveolar molding (PNAM) therapy using PSIO and J-hook appliance.

PNAM includes not only the reduction of the size of the intraoral alveolar cleft through the molding of the bony segments, but also the active molding and positioning of the surrounding soft tissues affected by the cleft, including the deformed soft tissue and cartilage in the cleft nose. ${ }^{4,5}$ This is accomplished through the use of a nasal stent that is based on the labial flange of a conventional oral molding plate and enters the nasal aperture, whereas in $\mathrm{J}$ hook appliance it takes support from the forehead [Figure 8]. The $\mathbf{J}$ hook stent provides support and gives shape to the nasal dome and alar cartilages without hindering the retention of PSIO appliance and better comfort.

\section{Case Report}

A 5-day-old male infant reported to the department with the patient's mother complaining of an opening over the lip and difficulty in feeding.

On clinical examination, the patient showed complete unilateral cleft lip, alveolus and palate deformity toward the right side. The infant weighed $2.8 \mathrm{kgs}$ on the initial visit [Figure 1].
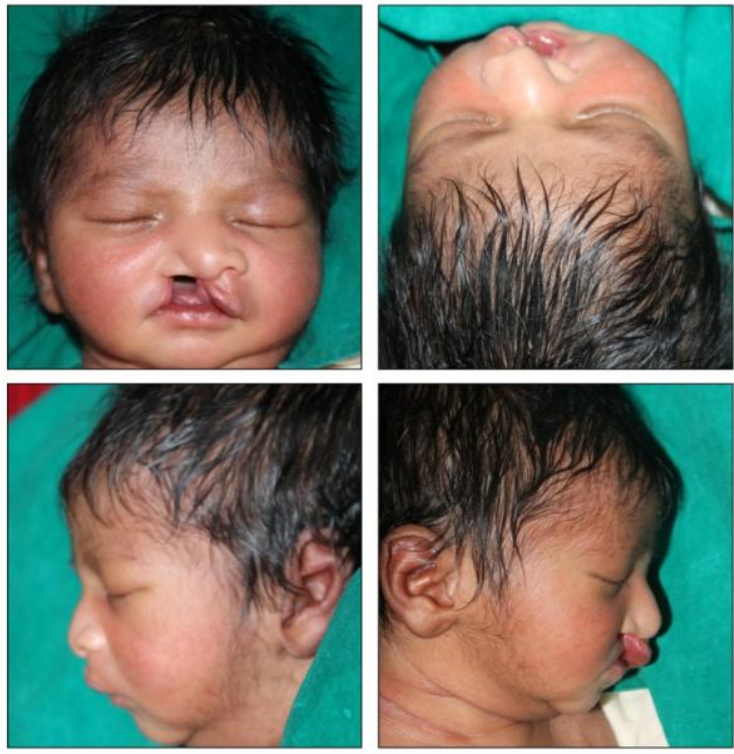

Figure 1: Pre NAM extraoral photographs $\left(5^{\text {th }}\right.$ day)

After a team evaluation of the case, the parents were explained about the procedure of PNAM including the potential benefits and possible complications. Most importantly the need for parental co-operation is highlighted during the first visit. Functional benefits to the infant such as better feeding without nasal regurgitation due to the presence of an intraoral acrylic plate were stressed upon. Complications like intraoral ulcerations due to rough areas on the plate or extra oral erythema on the cheeks due to improper tape removal were also explained. 
This was followed by an intraoral primary maxillary impression which was made using modeling wax sheet dipped and softened in mild warm water, primary cast was poured and a special tray was fabricated using acrylic resin to take the secondary impression.

On the same day, secondary impression of the intraoral cleft was made using an elastomeric material on the special tray. While making secondary impression, the baby was fully awake and without any anesthesia in his mother's lap with head facing downward. The infant was held face down to prevent the possible aspiration of regurgitated stomach contents. The mother cradled the infant securely around the chest and torso, supporting the head and neck while impression was obtained. The infant was made to cry during the impression-making procedure to prevent the blockage of airway. A finger motion was used to clear impression material posterior to the tray in order to prevent the infant from closing down on the tray, which would have also compromised the airway.

On the first visit itself, lip taping was done using Steri-Strip (3M Steri-Strip ${ }^{\mathrm{TM}}$ ). A base tape was placed and a hydrocolloid type bandage was placed over the cheeks since it serves as a barrier between the retention tapes and the cheeks to minimize tissue irritation. Instructions were given to parents about lip taping and were advised to continue lip taping for a week [Figure 2].

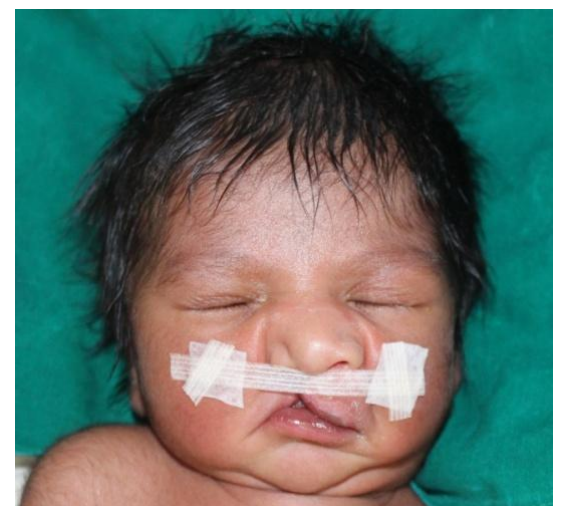

Figure 2: lip taping- $5^{\text {th }}$ day (first appointment)

Two orthokal stone casts were obtained one of which served as the working model and the other as study model. The alveolar cleft and any other undercut areas on the working model were blocked and auto polymerizing acrylic resin (DPI-RR Cold Cure, India) was used to fabricate a plate not more than $2 \mathrm{~mm}$ in thickness [Figure 5]. Traditional NAM requires serial modifications in the internal surfaces of the molding appliance with addition or deletion of material in certain areas to get desired shape of the alveolus and nose (Negative Sculpturing); these frequent adjustments create a burden for patients living far from a hospital. To minimize this burden, we followed the modified NAM technique with an active palatal plate where the cleft alveolus is free to approximate physiologically with the pressure exerted by the plate (Passive Molding). The plate was checked for smoothness and absence of any rough regions and a trial fit was undertaken in the infant's oral cavity on the second visit. Proper fit of the plate was evidenced by, the infant making attempts at sucking on the plate during the trial procedure. Proper positioning of the acrylic retentive button was crucial, the button has to be positioned so that it does not prevent lip approximation and vertically it should lie in between the upper and lower lips. The correct position is marked and the retentive button is attached to the intraoral plate using auto polymerizing acrylic resin [Figure 6]. The retentive button connects the intraoral plate to the extraoral elastic tape force system. 

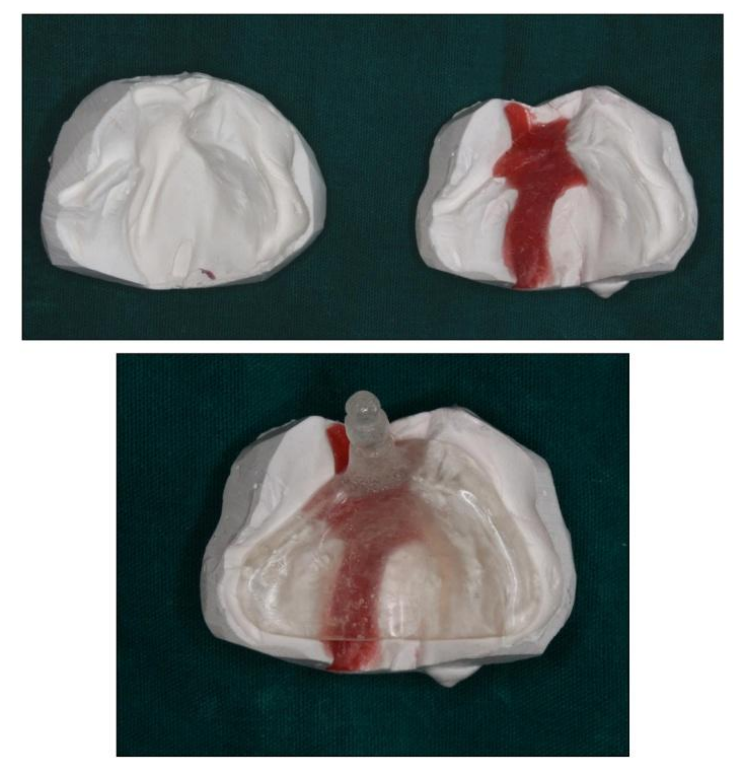

Figure 5: Fabrication of PSIO appliance
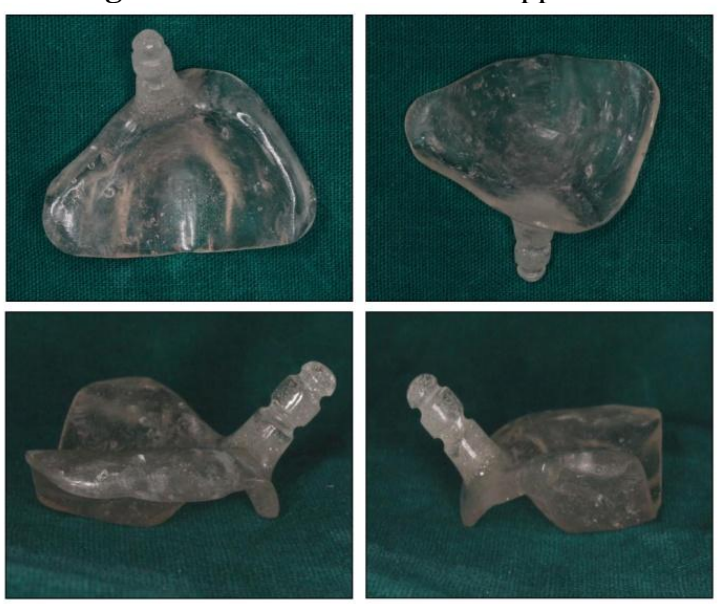

Figure 6: PSIO appliance

On the second appointment a well-finished and polished molding plate was inserted into the infant's mouth on the same day to promote feeding as that was one of the chief complaints of the infant's mother. Small red orthodontic elastics (0.25- inch diameter) were incorporated into the loops of thinner Steri- Strips that are folded over on themselves. The elastic band is placed over the retentive button, and the Steri-Strips were pulled and secured to the base tapes on the baby's cheeks [Figure 7]. Enough retentive force had been exerted when the elastics were pulled to twice their original length. The correct force vector on the retentive tapes and elastics were directed posteriorly and superiorly. The Steri-Strips and elastic bands were changed as and when necessary to allow continuous retention of the appliance. The base tapes remained in place for a longer period of time, while thin tapes required replacement several times. 

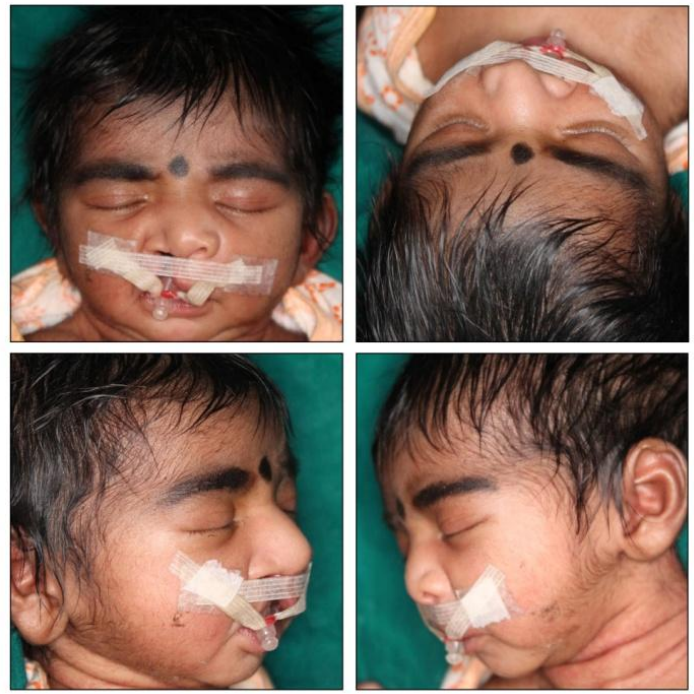

Figure 7: NAM plate delivery (PSIO appliance)

After 2 months, when the cleft gap has been reduced to approximately $5 \mathrm{~mm}$ unlike conventional nasal attachment stents a modification was given to enhance retention and to avoid bulkiness of the molding plate. The $\mathrm{J}$ hook appliance is a stent with a projection of acrylic supported by round stainless steel wire which was attached to the forehead [Figure 8]. Conventional nasal stent also exerts a reciprocal intraoral molding force against the alveolar segments hence this drawback was eliminated by using J-hook modification appliance. The J-hook nasal stent was inserted passively into the nostril and covered with a thin veneer of soft reliner to apply positive elastic pressure. The J-hook stent appliance was delivered and the phase of active nasal cartilage molding was started. This pressure aids to lift the collapsed nostril and in molding the nasal tissue.
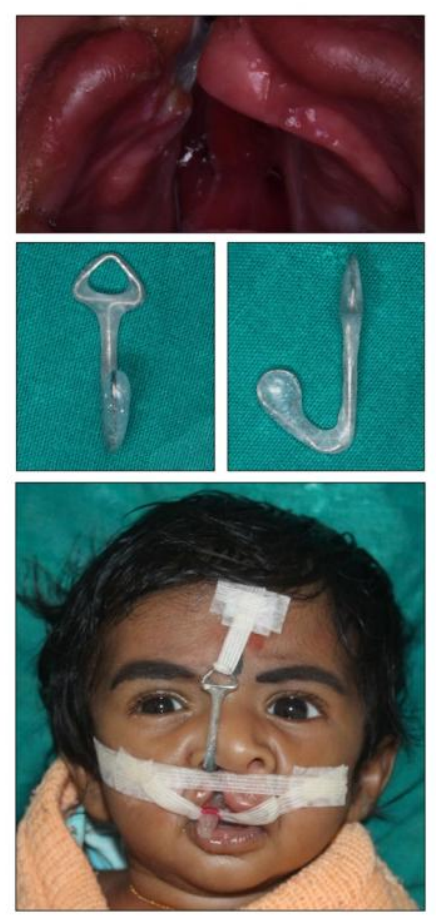

Figure 8: Fabrication and delivery of J-hook appliance

During follow-up visits, the molding plate as well as the nasal stent was modified by serial addition and subtraction of soft reliner to get the desired shape of nostril, ala of nose and to approximate the alveolar segment in a desired anatomy. On each appointment, the infant's weight was checked that showed a progressive good amount of increase 
which indicated that NAM plate improved feeding. At the end of NAM, there was reduction in the alveolar cleft from $14 \mathrm{~mm}$ to $3 \mathrm{~mm}$ [Figure 11] and the columella was lengthened and repositioned from an oblique position into an upright position, which resulted in improved nasal alar cartilage symmetry [Figure 4,9]. At the end of 5 months when the infant weighed $8.5 \mathrm{kgs}$, the contour of the nostril on the cleft side resembled the unaffected side.

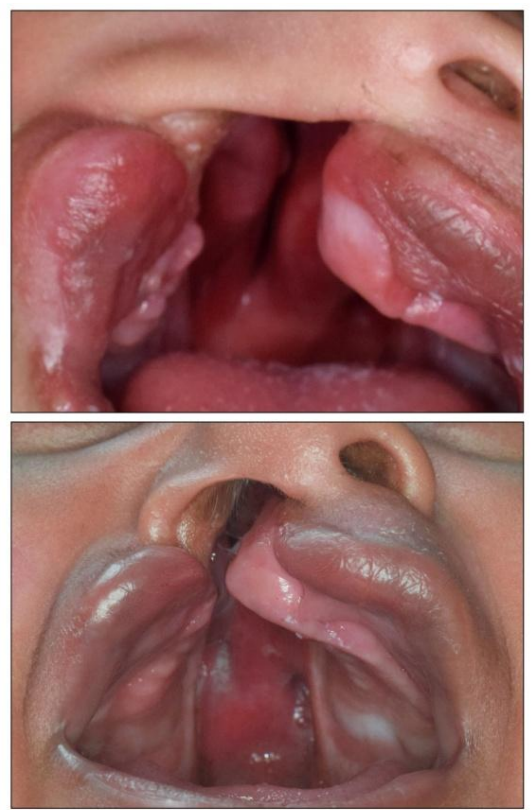

Figure 4: Pre and post orthopeadic cleft alveolus intraoral photographs
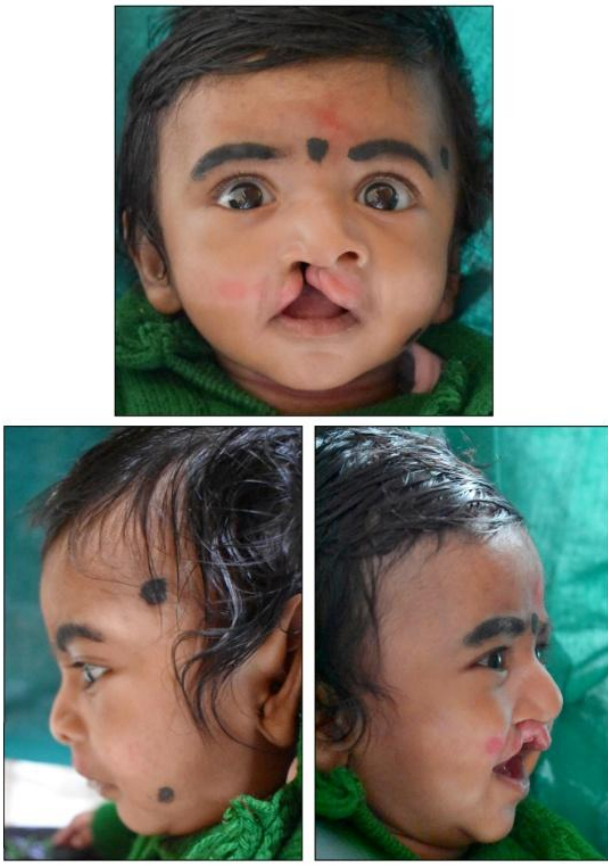

Figure 9: End of lip taping and PSIO-J hook appliance

The infant was evaluated a week prior to surgery by both the surgeon and the orthodontist for a final evaluation of the lips, alveolus and nasal position. No modifications were done on the appliance since it may introduce tissue irritation just prior to surgery. Following surgical repair of lip, the lip was taped for several weeks and the use of PSIO-J hook appliance was discontinued [Figure 10]. 


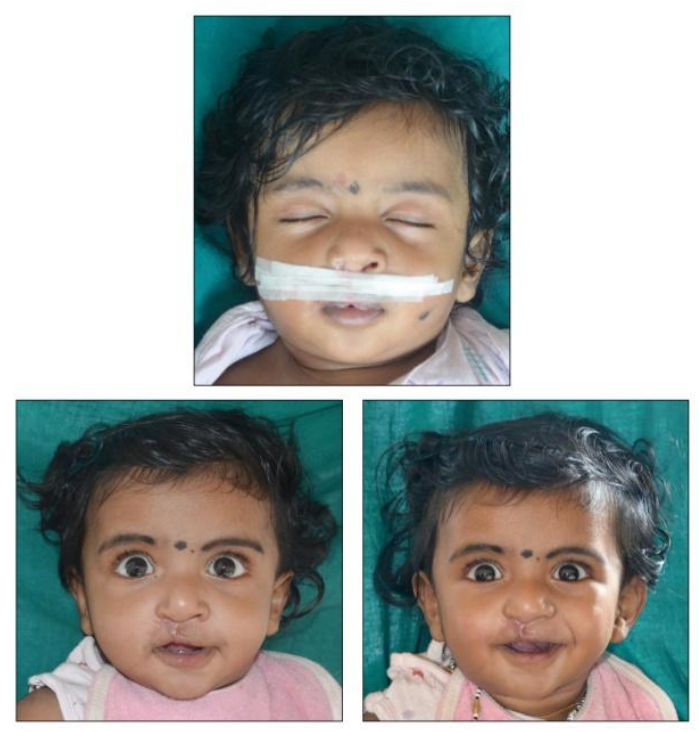

Figure 10: Post surgical photographs

\section{Results:-}

PNAM using PSIO-J hook appliance helped in reducing the extent of the deformity in all dimensions from pretreatment at the level of nose and lip. There was significant improvement in nasal tip projection, columella size on cleft side and position of lower lateral cartilages as well as approximation of the lip segments [Figure 3]. Additionally the alveolar defect was also reduced to a significant extent from pre NAM to post NAM [Figure 4,11]. The postoperative recovery was uneventful.
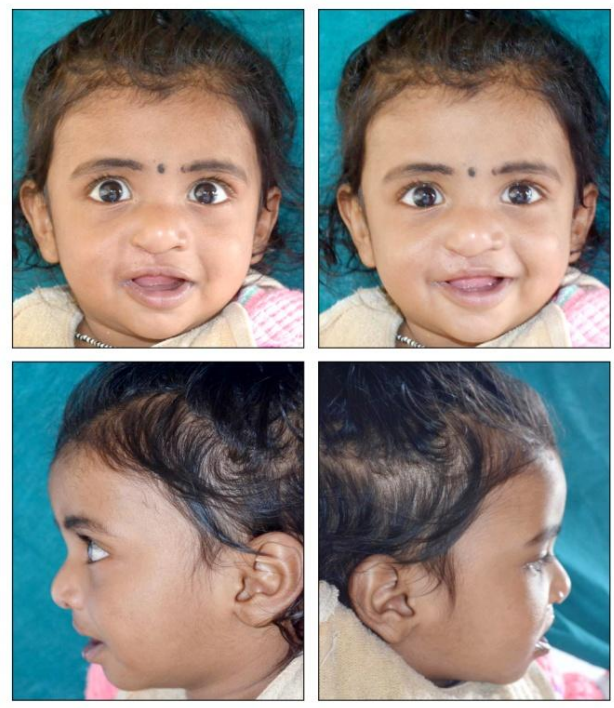

Figure 3: Post NAM extraoral photographs

\section{Discussion:-}

As success in NAM is highly dependent on the age of commencement of therapy and parental cooperation results may not be consistent. The role of parents in ensuring success of PNAM needs to be emphasized. ${ }^{4}$ Parents need to be motivated and explained the positive impact that PNAM can deliver prior to surgery, paving the way for easier and more aesthetic surgery. One of the contraindications to PNAM is lack of adequate compliance from parents in spite of repeated instructions. Increased age of the infant is another contraindication for PNAM as the plasticity of the cartilages begins to wane after the first two months of life. ${ }^{7}$ It is therefore important to have an aggressive referral system so that orthodontic intervention with PNAM can be initiated as early as possible. 
Management of the cleft deformity aims at achieving normal anatomy and function. The purpose of Presurgical infant orthopedic (PSIO) appliances is achieving a near to normal anatomic relationship of the affected structures significantly in reducing the size of clefts of the alveolus and hard palate prior to surgery. PSIO aids in reducing the extent of distortion, deviation and displacement of the affected structures. ${ }^{10}$ At present, there are three different PNAM techniques routinely used, namely Grayson's technique, Figueroa's technique, and Liou's technique. PSIO-J hook appliance is a combination of intraoral molding orthopaedic appliance with an additional extraoral anchoraged J-hook nasal stent. With nasal molding there is improvement in nasal morphology prior to repair, primarily the columella size and orientation, alar base and lower lateral cartilage position and nasal tip projection. PSIO-J hook appliance greatly enhances the retention and helps to avoid the unwanted intraoral reciprocal molding force which hinders the fusion of alveolus. Because the molding plate and the nasal stent are independent from each other, the PSIO-J hook appliance is easy to fabricate and use. The appliance can be fabricated during any spare time because an impression of the nasal region is not needed. Presurgical lip taping is standard in most infant maxillary orthopedic approaches and ensures that there is reduced tension at the surgical site after repair.

\section{Conclusion:-}

PSIO-J hook appliance greatly improves the retention of molding plate along with reducing the bulkiness compared to the conventional plate. The reciprocal intraoral molding force against the alveolar segments during conventional nasal stent was eliminated by using J-hook modification appliance. The pre-surgical nasoalveolar molding assisted primary reconstruction using PSIO-J hook appliance results is an overall improvement in the esthetics of the nasolabial complex in cleft conditions while minimizing the extent of surgery and the overall number of surgical procedures. PSIO-J hook appliance, when used prior to primary surgical lip repair will produce greater positive effect in the esthetics, psychological and social arena.

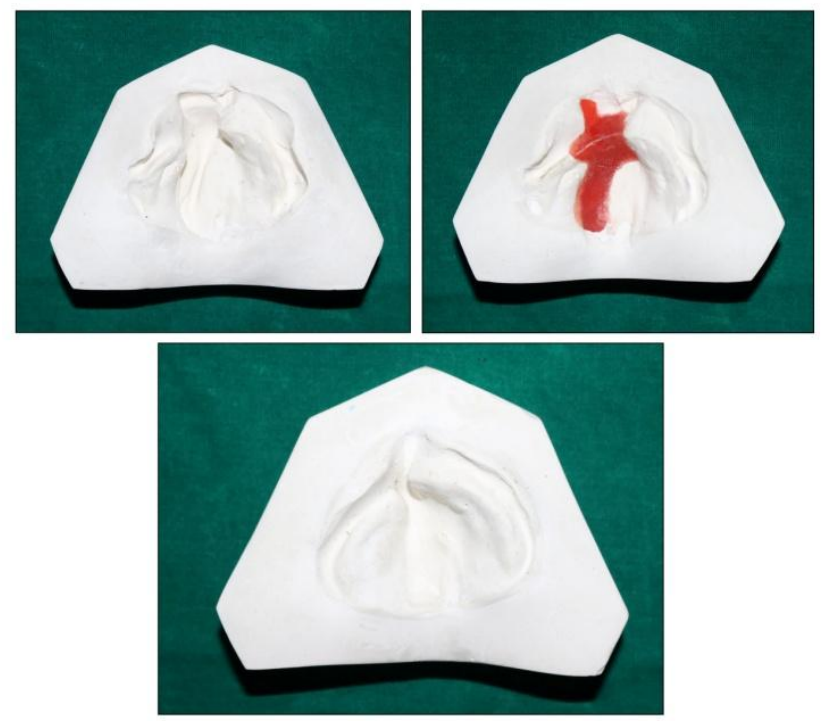

Figure 11: Pre NAM and Post NAM alveolar defect changes

\section{References:-}

1. Doneria D, Thakur S, Uppal A, Chauhan A. Figueroa modified presurgical nasoalveolar molding for cleft patients: A case series of three cases. Journal of Cleft Lip Palate and Craniofacial Anomalies. 2018 Jul 1;5(2):119.

2. Grayson BH, Cutting CB. Presurgical nasoalveolar orthopedic molding in primary correction of the nose, lip, and alveolus of infants born with unilateral and bilateral clefts. The Cleft palate-craniofacial journal. 2001 May;38(3):193-8.

3. Doruk C, Kiliç B. Extraoral nasal molding in a newborn with unilateral cleft lip and palate: a case report. The Cleft palate-craniofacial journal. 2005 Nov;42(6):699-702.

4. Mathew A, Muddaiah S, Subrahmanya J, Somaiah S, Shetty B, Reddy G. Presurgical nasoalveolar molding in a 4-day-old infant with unilateral cleft lip, alveolus, and palate deformity. APOS Trends in Orthodontics. 2018 Oct $1 ; 8(4): 225-$. 
5. Barillas I, Dec W, Warren SM, Grayson BH. Nasoalveolar molding improves long-term nasal symmetry in complete unilateral cleft lip-cleft palate patients. Plastic and reconstructive surgery. 2009 Mar 1;123(3):1002-6.

6. Radhakrishnan V, Sabarinath VP, Thombare P, Hazarey PV, Bonde R, Sheorain A. Presurgical nasoalveolar molding assisted primary reconstruction in complete unilateral cleft lip palate infants. Journal of Clinical Pediatric Dentistry. 2010 Apr 1;34(3):267-74.

7. Mishra B, Singh AK, Zaidi J, Singh GK, Agrawal R, Kumar V. Presurgical nasoalveolar molding for correction of cleft lip nasal deformity: experience from northern India. Eplasty. 2010;10.

8. Liang Z, Yao J, Chen PK, Zheng C, Yang J. Effect of presurgical nasoalveolar molding on nasal symmetry in unilateral complete cleft lip/palate patients after primary cheiloplasty without concomitant nasal cartilage dissection: Early childhood evaluation. The Cleft Palate-Craniofacial Journal. 2018 Aug;55(7):935-40.

9. Koya S, Shetty S, Husain A, Khader M. Presurgical nasoalveolar molding therapy using Figueroa's NAM technique in unilateral cleft lip and palate patients: a preliminary study. Journal of Clinical Pediatric Dentistry. 2016 Jun;40(5):410-6.

10. Premkumar S, Kunthavai CR, Tovani-Palone MR. Clinical application of customized presurgical nasoalveolar molding for the treatment of unilateral complete cleft lip and alveolus: case report. Electronic Journal of General Medicine. 2018 Jan 1;15(5).

11. Kornbluth M, Campbell RE, Daskalogiannakis J, Ross EJ, Glick PH, Russell KA, Doucet JC, Hathaway RR, Long Jr RE, Sitzman TJ. Active Presurgical Infant Orthopedics for Unilateral Cleft Lip and Palate: Intercenter Outcome Comparison of Latham, Modified McNeil, and Nasoalveolar Molding. The Cleft Palate-Craniofacial Journal. 2018 May;55(5):639-48.

12. Nagraj N, Nagarjuna M, Desai AK, Gandedkar N, Jayade B, Gopalakrishnan K. Double-loop technique using titanium molybdenum alloy wire for fabrication of nasal stents in nasoalveolar molding therapy for cleft lip and palate patients. The Cleft Palate-Craniofacial Journal. 2015 Mar;52(2):246-9.

13. Titiz S, Aras A. Presurgical orthopedic treatment using modified nostril retainers in patients with unilateral cleft lip with or without cleft palate. Journal of Craniofacial Surgery. 2017 Sep 1;28(6):1570-2.

14. Jodeh DS, Ruso S, Feldman R, Ruas E, Rottgers SA. Clinical Outcomes Utilizing a "Modified Latham" Appliance for Presurgical Infant Orthopedics in Patients With Unilateral Complete Cleft Lip and Palate. The Cleft Palate-Craniofacial Journal. 2018 Dec 9:1055665618816892.

15. Chen YF, Liao YF. A modified nasoalveolar molding technique for correction of unilateral cleft nose deformity. Journal of Cranio-Maxillofacial Surgery. 2015 Dec 1;43(10):2100-5. 